\title{
CMS detector tracking performance in Run-II
}

\author{
Erica Brondolin* \\ Austrian Academy of Sciences (AT) \\ E-mail: erica.brondolinecern.ch
}

Since the start of Run-II in June 2015, LHC has delivered pp collisions at a center of mass energy of $13 \mathrm{TeV}$ and with a bunch time separation of $25 \mathrm{~ns}$. On avarage, more than 25 inelastic collisions are superimposed on the event of interest. Under these new conditions, the CMS collaboration has re-calibrated and verified the performance of the whole detector. In particular, the CMS tracking performance has been measured both directly and indirectly. Direct measurements are, among others, the beam spot determination, the vertex resolution and the muon reconstruction efficiency with the tag and probe technique. An indirect assessment can be given by the pion reconstruction efficiency and the low-mass resonance parameters as a function of different single track kinematics.

38th International Conference on High Energy Physics 3-10 August 2016

Chicago, USA

${ }^{*}$ Speaker. 


\section{Introduction}

In order to cope with the new conditions in Run-II, some developments have been made in different aspects of the tracking procedure on top of the already high-quality software developed for Run-I [1,2]. In order to check the tracking performance and to understand in as much detail as possible the Monte Carlo (MC) simulations, both direct and indirect measurements have been used in Run-II.

\section{Direct Measurements}

Measurements of the beam spot parameters and the vertex resolution, along with the muon reconstruction efficiency, are some examples of direct measurements of the tracking performance.

The beam spot is obtained by continuous monitoring of the luminous region where the two LHC beams collide inside CMS. Its measurement plays a crucial role for both the trigger selection and the event reconstruction, where its position is used as a constraint for the track fitting when the primary vertices of the event are not yet determined, and for the primary vertex reconstruction. The beam spot parameters are estimated with two different methods for each luminosity section ${ }^{1}$. The first method is based on an unbinned 3-Gaussian likelihood fit to the 3-D distribution of the reconstructed primary vertices of the events, while the second one extrapolates the beam spot parameters using an iterative $\chi^{2}$ fit to the joint distribution of the impact parameter and the azimuthal angle of the reconstructed tracks. The two methods give consistent results with those expected from the LHC tuning for Run-II : the beam spot transverse width has been measured around $14 \mu \mathrm{m}$ along $x$ and $12.5 \mu \mathrm{m}$ along $y$, while the longitudinal width drops from around $4 \mathrm{~cm}$ to about $3 \mathrm{~cm}$ during the fill, depending on the beam energy losses due to synchrotron radiation at $6.5 \mathrm{TeV}$.

The vertex resolution is measured taking into account primary vertices with more than four tracks. The tracks associated to the primary vertex are sorted in descending order of transverse momentum and then grouped in pairs. Two sets of tracks are then created using a random assignment of the pairs, so that the two sets have same kinematic properties, and fitted independently with the adaptive vertex fitter. The primary vertex resolution is estimated as the standard deviation of the Gaussian fit to the difference of the re-fitted vertex positions, divided by $\sqrt{2}$. The results for Run-II data are shown in Figure 1(a). For primary vertices with a sum of track $p_{\mathrm{T}}$ larger than $100 \mathrm{GeV}$, the transverse resolution is better than $14 \mu \mathrm{m}$, and the longitudinal one is better than $19 \mu \mathrm{m}$.

The last direct method described in this paper to measure the tracking performance is the tag and probe method. It allows to assess the performance of $\mathrm{MC}$ data by comparing the particle reconstruction efficiencies in real data with the efficiency predicted by the MC simulation. In the first step, the $Z$ particle resonance is reconstructed as pairs of muons, one of which has passed a tight identification (the so-called tag), while the other one has passed a loose identification (the so-called probe). In the specific context of this analysis, the tag is defined as a muon with $p_{\mathrm{T}}$ more than $15 \mathrm{GeV}$, associated to one leg of the resonance, while the probe can be any stand-alone muon ${ }^{2}$ with more than one valid hit. Probes are then divided into two categories, passing and failing, depending on whether or not they can be matched to at least one track. The matching uses the

\footnotetext{
${ }^{1}$ Every luminosity section lasts about 23 seconds.

${ }^{2} \mathrm{~A}$ stand-alone muon is a muon recontructed using just the muon chambers information.
} 


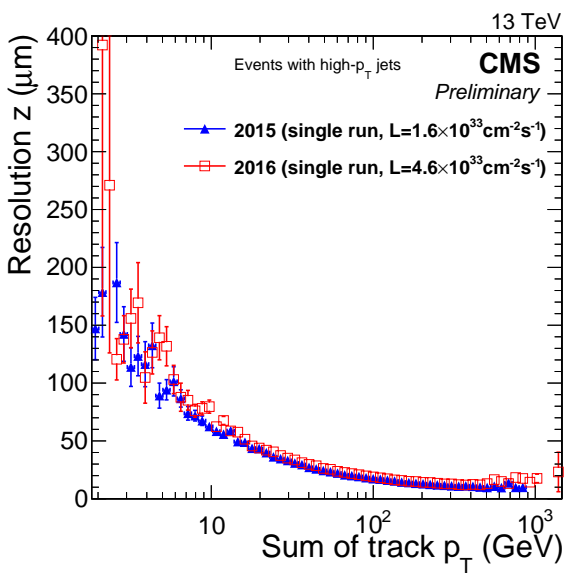

(a)

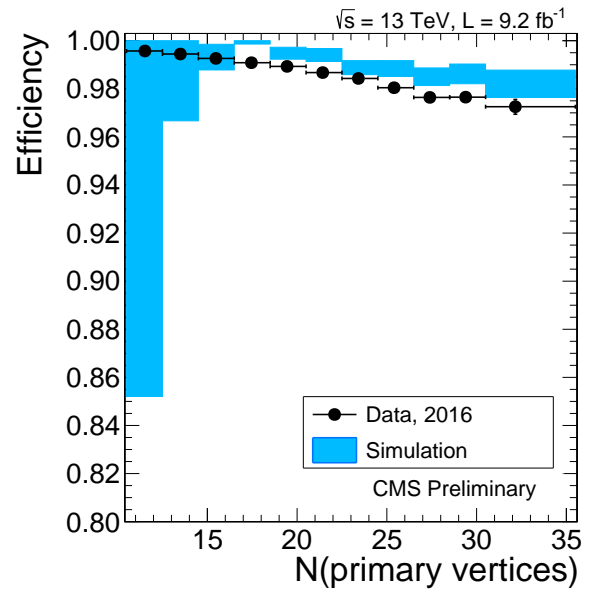

(b)

Figure 1: (a) Comparison of the primary vertex resolution in $z$ vs the sum of track $p_{\mathrm{T}}$ of the associated tracks for 2015 (blue triangles) and 2016 data (red rectangles) [3]. (b) Data (black dots) and simulation (light blue rectangles) tracking efficiency for muons coming from the $\mathrm{Z}$ decay as a function of the number of primary vertices [3].

size of the cone in space drawn around the stand-alone muon. In the last step, the invariant mass spectrum of the pair tag/probe is fitted, and the muon tracking efficiency is computed as the ratio of the number of the passing probes over the total number of probes in the sample. Figure 1(b) shows the muon tracking efficiency for 2016 data as a function of the number of primary vertices. The efficiency is between $99.5 \%$ and $97 \%$ with a strong dependence on the number of primary vertices. The agreement between data and simulation reconstruction efficiency is very good and the entire set of results can be found in [3].

\section{Indirect Measurements}

The tag and probe method cannot be used for finding the reconstruction efficiency of hadrons because of decays in flight and nuclear interactions within the detector. In order to measure the tracking efficiency in this case and compare it with the MC, indirect methods are used.

The relative tracking efficiency for charged pions is computed using the ratio of neutral charmmeson decays to final states of four or two charged particles. Two decay chains from $D^{+} \rightarrow D_{0} \pi_{\text {slow }}$ are reconstructed in data and MC: the "four-body final state" $D_{0} \rightarrow K^{-} \pi^{+} \pi^{-} \pi^{+}(K 3 \pi)$ and the "two-body final state" $D_{0} \rightarrow K^{-} \pi^{+}(K \pi)$. Assuming that the decay chains are well represented in the Monte Carlo samples, the ratio $R$ between their branching ratio can be measured using the formula $R=\frac{N_{K 3 \pi}}{N_{K \pi}} \frac{\varepsilon_{K \pi}}{\varepsilon_{K 3 \pi}}$, where $N$ is the number of events and $\varepsilon$ is the selection efficiency in the respective decay chain. The pion relative efficiency is then found comparing $R$ with the nominal value in the PDG $R_{\text {PDG }}=2.08 \pm 0.05$ [5]. Figure 2(a) shows the good agreement between $R$ and $R_{\text {PDG }}$, which implies that the efficiency computed using the MC correctly reproduces the 2015 data in all three pseudorapidity ranges [4]. 


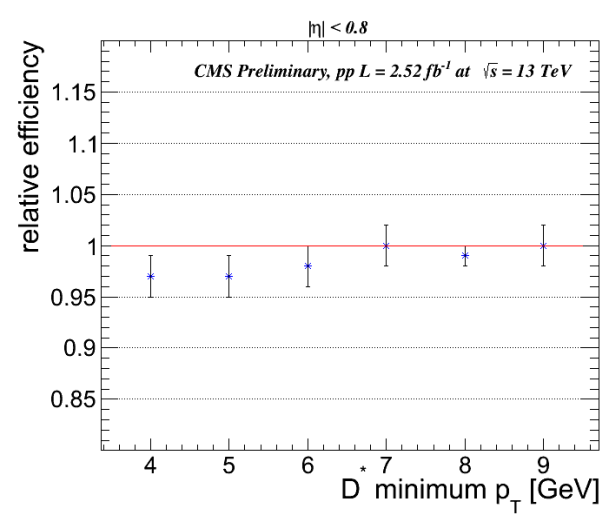

(a)

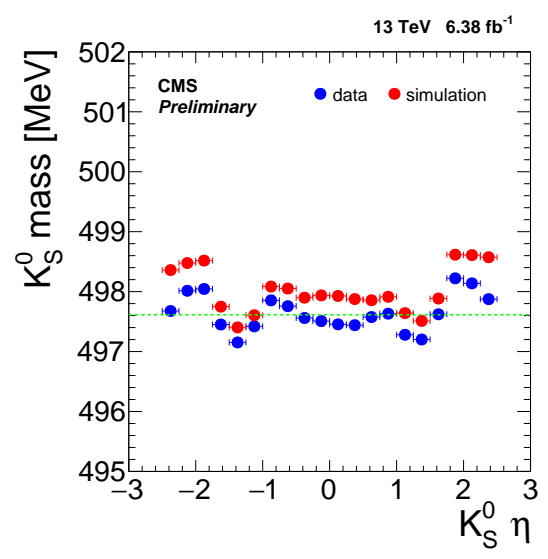

(b)

Figure 2: (a) The ratio of relative tracking efficiencies for charged pions, as a function of the $D^{*}$ minimum $p_{\mathrm{T}}$ in the barrel region [4]. (b) $K_{\mathrm{S}}$ invariant mass as a function of the pseudorapidity for data (blue points) and simulation (red points) [3].

Another indirect measurement uses low-mass resonances such as $K_{\mathrm{S}} \rightarrow \pi^{+} \pi^{-}$and $\Lambda_{0} \rightarrow$ $\pi^{ \pm} p^{\mp}$. These particles have a narrow intrinsic width mainly dominated by the limited knowledge and modeling of the detector material, the magnetic field and the reconstruction algorithms used to fit the track trajectory. If these effects are fully understood, the systematic uncertainties which affect the momentum resolution and scale of charged tracks can be determined with good precision and the modeling of the detector and the track reconstruction can be improved. A study of the average mass as a function of different single track kinematics both in simulation and data has been carried out, and the entire set of results can be found in [3]. In Figure 2(b), the invariant mass of the $K_{\mathrm{S}}$ particle as a function of the pseudorapidity is presented for data and simulation. The shape is well described by the simulation and data and simulation agree at the level of few per mille in the tracker volume. The same conclusions apply also for the $\Lambda_{0}$ particle results.

\section{References}

[1] CMS Collaboration, Description and performance of track and primary-vertex reconstruction with the CMS tracker, JINST 9 (2014) P10009, doi:10.1088/1748-0221/9/100/P10009.

[2] G. Cerati, Tracking and vertexing algorithms at high pileup, Vertex 2014 Conference Report, https://cds.cern.ch/record/1966040/files/CR2014_345.pdf

[3] CMS Collaboration, CMS Tracking POG Performance Plots 2016, https: //twiki.cern.ch/twiki/bin/view/CMSPublic/TrackingPOGPlotsICHEP2016

[4] CMS Collaboration, CMS Tracking POG Performance Plots for EPS 2015, https: //twiki.cern.ch/twiki/bin/view/CMSPublic/TrackingPOGPlotsEPS2015

[5] Particle Data Group collaboration, Review of Particle Physics, doi:10.1088/1674-1137/38/9/090001 